\title{
The CLIPR remote laboratory microprocessor system: Development and applications
}

\author{
DANIEL E. BAILEY, WAYNE H. WARD, TERRY L. SPEAR, \\ RONALD L. LEATHERMAN, JOANNE L. WAITE, and THOMAS W. CHRISTIAN \\ Computer Laboratory for Instruction in Psychological Research \\ University of Colorado, Boulder, Colorado 80309
}

The introduction of microprocessor technology into the centralized experiment automation facilities of CLIPR separates data processing functions and real-time functions. Three examples of real-time intelligent terminals are described. The implementation process, principles, and examples for using microprocessor technology in this way is described in some detail.

The development of the Computer Laboratory for Instruction in Psychological Research (CLIPR) at the University of Colorado is documented in previous papers of this series (Bailey, 1972, 1973; Polson, 1978) and elsewhere (Bailey \& Polson, 1975). This paper presents some detail of the "new look" at CLIPR: remote laboratory microcomputer systems.

First, we discuss the background of the development of microcomputer systems at CLIPR. Then, we illustrate the concept of the remote laboratory microcomputer system with three specific systems in operation or in development at CLIPR. Next, we describe the hardware and software questions that are essential issues in planning such systems. Finally, we discuss some of the implementation details encountered in making the systems work.

\section{CLIPR BACKGROUND}

CLIPR has been an experiment in centralization of computer-automation facilities within a department of psychology, the results of which have been positive. It is possible to centralize and to achieve a high degree of success in automating a diverse spectrum of research programs. Although the hardware costs of a central facility are generally greater than "stand-alone" "oneperson" laboratory computers, the payoff is greater, in terms of total efficiency and productivity. Also, if the costs of automating several laboratories separately

Portions of the work reported in this paper were supported by Grants NSF GJ-453 (D. E. Bailey, principal investigator), NIDA DA01467 (P. M. Groves, principal investigator), NSF BNS 76-04652 (L. O. Harvey, Jr., principal investigator), and PHS 5507BR07013-12 (D. E. Bailey and L. O. Harvey, Jr., principal investigators). Requests for reprints or additional information should be directed to D. E. Bailey, E318 Muenzinger, CLIPR, University of Colorado, Boulder, Colorado 80309. Portions of the video equipment in the system described here were made available through a gift from Colorado Video, Inc., of Boulder, Colorado. We wish to express our gratitude to CVI, and especially to Glen Southworth and Wyndham Hannaway, for their generosity and assistance. are aggregated, the "break-even" point is reached with a relatively small number of stand-alone systems. The cost factor for stand-alone systems is greatly magnified if several systems are independently staffed, a situation often forced by nonintegrated hardware and software development.

Technology is changing so fast that different considerations appear to be active now than those in effect when CLIPR began operations: Large-scale integration and microcomputer technologies have redefined some of the central issues in automating psychological research.

This paper covers a wide range of topics and, consequently, deals with many of them at a superficial level. However, it serves to illustrate the new technology and to touch on issues that are critical to its development more generally.

\section{REALTIME INTELLIGENT TERMINAL SYSTEMS}

As experiment automation expanded into the laboratories in the department of psychology at the University of Colorado, the strength of the shared central computer became apparent. We began to execute simultaneously several programs with large numbers of subjects. It is not uncommon to have a dozen asynchronous real-time processes being executed on the CLIPR computer system. The ready availability of realtime computer-automation services attracted numerous users. The success of the CLIPR experiment began to lead to demands that we could not gracefully meet, and we began to discover some of the boundary conditions previously lurking out of sight. The most significant limitation in the hardware technology (1969 vintage) was memory space. The "fixed partition." memory scheme imposed a severe upper limit on the number of separate real-time processes that could run simultaneously. On the other hand, the cost and technical difficulty of replacing the fixed memory scheme with more dynamic memory utilization loomed prohibitive. Furthermore, certain processes being introduced into 
the mix of applications were highly demanding of residency time, requiring perhaps many memorypartition hours for a single experimental data point. Also, we began to encounter high data rate experiments with periodic "real-time" compute-bound processing of the data. Both the length of time such programs would be resident in a central memory partition and the amount of CPU time utilized posed potential problems for the integrity of the multitask environment of the centralized system. We were principally concerned about resource (particularly memory) allocation for long-term run-time programs, central processor cycle availability, and associated problems of data accuracy (e.g., loss of real-time clock ticks) and data integrity (e.g., loss of ability to notice events in the laboratory).

We had several alternatives and options. Replacement of the central computer system with a larger system would satisfy immediate demands and, perhaps, provide a long-term solution. Financing was not available for this option. On the other hand, abandonment of the concept of centralized facilities and acquisition of separate computer systems for each project was rejected as incompatible with the lessons learned in CUPR.

We adopted a compromise solution made possible by the new microprocessor technology: Extend to the laboratory certain functions of the real-time tasks currently residing in memory partitions of the centralized facility. We proceeded to put real-time functions in remote terminals. This developed in CLIPR as a remote laboratory microcomputer system: a real-time intelligent terminal (RIT) within the real-time intelligent terminal system (RITS).

The basic concept of RITS is the separation of the functions performed in laboratory automation. Data processing functions, including programming, file maintenance, analysis, and so on, are separated from the real-time functions of instrument control, measurement, and data collection. Support facilities normally required for reasonable program development, computer operations, and data handling are located on a central or host computer. Experiment execution with instrument control, data collection, measurement, and so on, are located in individual RITs.

In the following, reference is made to specific microprocessors, jargon is used without definition, and assumptions are made about the knowledge of the reader in areas of microprocessor technology. For the reader who is not familiar with all of these things, we recommand the following texts: Barma and Porat (1976), Bibbero (1977), Osborne (1976a, 1976b), and Soucek (1976).

\section{THE CLIPR NETWORK}

Polson (1978) reported work in progress at CLIPR toward development of a network to support multiple RITs in a local environment. However, for purposes of completion and cogency of this paper, some discussion of the development of communications between the host computer and the RIT is required here.

The introduction of the term "host" is intended to suggest that a central laboratory computer (with real-time clock, interrupts, etc.) is not a necessary component in the use of RIT systems in laboratory automation. In general, within practical limitations, any computer having the capability of communicating to a RIT may serve as the host. The primary limiting factors are communication data rates, host responsiveness, and "data processing" facilities of the host.

Data rates that must be supported by the host for utilization by a RIT depend on the RIT configuration, the tasks to be performed by the RIT, and so on. If the data rates are not supported in the communications systems of a computer system, then it cannot serve as the host.

If the host computer is incapable of responding to requests for service from a RIT within the time limits imposed by the RIT and the application, then the system will fail due to inadequacies in the host. In networked or timeshared systems, one must be concerned about the capability of the host to deal with the number of RITs looking to the host for services.

The host must support sufficient software, programming language processors, file systems, editors, and so on, not also available in the RIT. Otherwise, the system is inadequate to the task. Assuming certain minimal requirements are met by both the host and the RIT, our focus is primarily on the two distal parts of the network: the host and the RIT. The network ideally will be transparent to the user of the RIT.

\section{SPECIFIC APPLICATIONS SYSTEMS}

Three specific RITs are presented as examples. Certain features of these application systems are used as points of reference in later sections.

\section{Slide Projector Driver}

This system is designed to collect two- and threeway comparison similarity data. The objects to be compared are images on photographic slides, which are projected by random-access slide projectors. One, two, or three slides may be presented simultaneously. The subject makes judgments about the similarities among the stimuli and indicates his decisions by pressing one of several pushbuttons. Other uses can easily be imagined. The system also measures response latencies. Programmed control of the projectors includes selection of slides, shutter control, and timing. Warning and feedback lamps are also under program control. As experimental trials are executed, the system can transmit response and latency data to the host system. Figure 1 is a block diagram of the physical and logical arrangement of this system. 


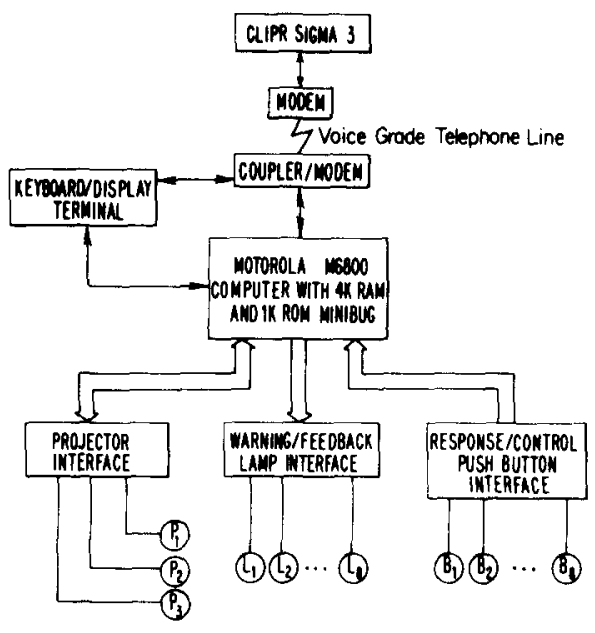

Figure 1. Slide projector driver configuration.

The RIT is interfaced to the host computer over voice-grade telephone lines by way of an acoustic coupler at the experiment site. A standard serial communications interface is incorporated into the RIT. Vendor-supplied modem and telephone interfaces are provided at the central computer facility.

The RIT is a modest configuration of a Motorola 6800 microprocessor (see Motorola Semiconductor Products, Inc., 1975, 1976a, 1976b) with 4K static RAM and MINIBUG ROM. Interfacing to the experimental devices is accomplished using commercially available peripheral interface adapters (PIAs), which are parallel interface chips, for both input and output. A 16-bit free-running clock with millisecond resolution is provided.

When the RIT is powered up initially, the MINIBUG routine is in control. Pressing the RESET button causes MINIBUG to send a predefined set of characters over the serial communication line to the host. Upon receiving this signal, the host sends a command to the RIT, causing the MINIBUG to begin accepting and storing (in RAM) the code constituting the RIT experiment program. The host then reads the program from the host file and sends it to the RIT. Finally, the host sends a command that causes MINIBUG to transfer control to the experiment control program.

The RIT is then ready to execute functions and make reports. The functions and reports to be executed are defined previously by the experimenter and processed into a series of codes that are interpretable by the control program. An ordered set of function codes and their parameters are referred to as a trial. The experimenter defines an experiment as a sequence of trials and submits the sequence to a translator on the host processor. The output from this translation is a series of control codes (and associated parameters) recognizable by the RIT control program. One trial at a time is transmitted to the RIT for interpretation by the control program. A trial may be arbitrarily long, limited only by the amount of RAM in the RIT. The experiment also may be arbitrarily long, limited only by storage space available on the host file system.

Reports of event and latency data are sent to the host during execution of an experiment as specified by report functions included in the trial definitions. These data are stored in a file allocated for that purpose on the host.

After the last function in a trial has been executed, an end-of-trial report is sent to the host and stored on the report file. The next trial is then sent to the RIT, and the entire process is repeated until the experiment code file has been exhausted.

The control program was written by CLIPR staff programmers and is available for experimenter use as a permanent feature in the CLIPR real-time library.

It is not necessary to include a keyboard/display terminal in this system, although such a device is shown in Figure 1. However, terminal functions have been incorporated to display experiment status and abort experiments.

Software development consisted of four separate projects. First, a cross-assembler on the CLIPR Sigma 3 was installed, so that assembly language programs could be prepared for the 6800 . The assembler produces absolute executable code for the microprocessor and stores it on a Sigma 3 disk file. Second, the interaction between the Sigma 3 and the RIT is conducted by way of a real-time FORTRAN program on the Sigma 3. The program consists primarily of a simple communication link between the microprocessor and the Sigma 3 files. Third, we designed and wrote a translator to convert the experiment functions and trials into codes that are used by the microprocessor control program in running an experiment. The output of the translator is stored on a file on the Sigma 3. Finally, using the cross-assembler, we wrote the 6800 experiment control program that interprets the control and report codes, reads the clock, and executes operations dictated by the control and report codes.

\section{Video Digitizer System}

This system is an interactive interface of a video digitizer/display system (Colorado Video, Inc., 1977a, 1977b) and the CLIPR Sigma 3. It has a wide variety of experimental applications, one of which is to provide digitized visual images suitable for Fourier analysis. An experimenter may thus obtain a two-dimensional Fourier transform of a stimulus to be used in vision experiments.

The system provides the facility to digitize a video image and store the information in the host computer or in RAM built into the display system. It also allows images stored on the host system to be transmitted to the video RAM and displayed on a video monitor. The hardware configuration is shown in Figure 2. The microprocessor system (Motorola 6800 ) has $4 \mathrm{~K}$ by tes of static RAM, a MINIBUG ROM, and a 1K EPROM. The 


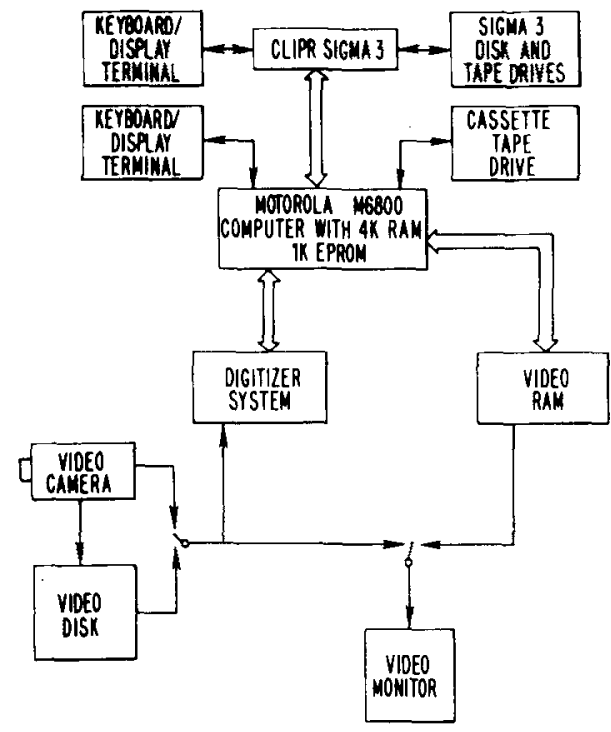

Figure 2. Video digitizer system configuration.

code for the digitizing system is stored on the EPROM. The user causes either the digitizer routine or MINIBUG to be in control by setting a switch. If the switch is set to the EPROM, the digitizer functions are ready to be executed. If the switch is set to MINIBUG, the user may enter routines to be executed. A cassette system and keyboard/display terminal interfaced to the RIT are proved for this purpose. To interface the RIT to the video devices, we used PIAs with memory-mapped I/O.

The video input to the system initially comes from a high-quality video camera. A video disk, which is a part of the video system, allows the user to store a single-frame input from the camera. Digitizer inputs may come from either the camera or the video disk. The video monitor may display either the camera/disk image or the video RAM image.

The RIT is programmed to execute a specific set of functions and acts as a slave to the host machine. The functions available allow the user to define a partition (window) of the entire digitizer space. This allows the user to digitize only that part of the frame that is of interest, minimizing data transfer and storage. The window also applies to the video RAM. By moving the window (and changing size if desired), the user may display different images on different portions of the monitor screen simultaneously.

The user requests function execution by entering a mnemonic and parameters via the keyboard/display terminal. The mnemonic is transmitted to the host over a standard serial communications line. The host decodes the mnemonic and sends a function command to the RIT over parallel lines. The host then continues processing other tasks until it receives a response on the parallel I/O lines from the RIT, indicating that the function has been executed. Data resulting from function execution are stored in the microprocessor memory. When the RIT has completed execution of a function, it sends an interrupt signal to the host, indicating that it is through. When the host responds to the interrupt, the data are sent to the host over the parallel lines and stored in a file on the host. Data stored on host files may also be transmitted to the RIT, stored in RIT memory, and from there written out to the video RAM.

Two programs were developed to implement this system. The host program, which runs on the Sigma 3, handles keyboard/display terminal I/O, decodes mnemonic functions, handles $\mathrm{I} / \mathrm{O}$ to the RIT, and executes the appropriate file management routines to store and retrieve data. The RIT program was developed using the 6800 cross-assembler on the host machine. After the 6800 program was fully developed and determined to be functioning properly, it was burned into an EPROM, which was then placed in the RIT memory board. Development and testing phases involved use of a RAM memory for the control program.

The RIT has few data manipulation functions but serves primarily to interface the host to the video devices. This method of interfacing significantly reduces the load on the host computer. CLIPR is in the process of replacing this system with one based on the Texas Instruments 9900 (see below) microprocessor. The 9900 system will have many more local data manipulation capabilities, such as contrast enhancement, Fourier analysis, and so on.

\section{Neuron Activity Monitor}

The neuron activity monitor performs real-time data collection and analysis of electrical activity of brain cells of laboratory animals. Data are collected from a microelectrode implanted in a laboratory rat's brain. The analog signal from the electrode is processed by a spike-detector circuit and by an A/D converter to produce computer-usable representations of the data. This system is currently still under development, but we speak of it as existing to simplify the description.

Data collected include spikes per minute, occurrence times of spikes, and a digitized representation of the full waveform at prescribed intervals. Analysis of the data during the course of the recording session includes tabling the current spikes per minute, the session average spikes per minute, spike interarrival time histogram, and histograms (coarse and fine resolution) representing the autocorrelation of spike occurrence times. The experimenter is allowed to specify frequencies with which the current state of the data analysis are saved.

A monitor displays the current status of the experiment during the course of an experimental session. The status display contains current values of experiment control parameters and values of the current data analysis, including graphic display of interarrival and autocorrelation histograms.

The hardware configuration for this system is shown 


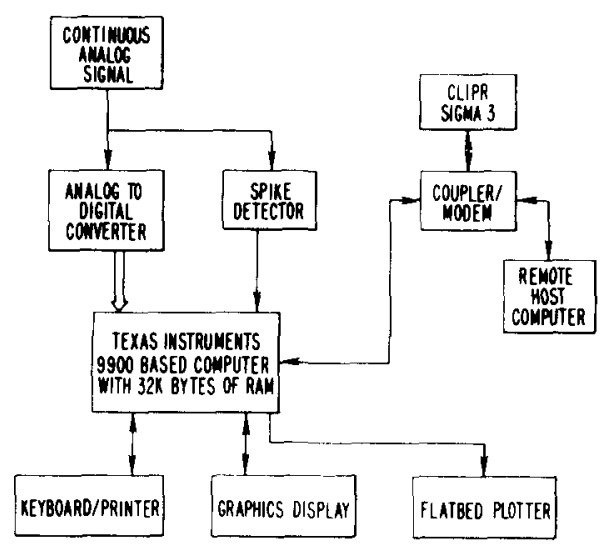

Figure 3. Neuron activity monitor configuration.

in Figure 3. The microcomputer contains a Texas Instruments 9900 microprocessor (Texas Instruments, Inc., 1976a, 1976b, 1976c) on a custom-built CPU module. The remaining modules of the main frame, memory, peripheral interfaces, and so on, are implemented almost entirely by widely available off-the-shelf board-level components for the S100 bus (Goble, 1977; Morrow \& Fullmer, 1978). The current configuration has $32 \mathrm{~K}$ bytes of 250 -nanosec RAM and is expandable to $64 \mathrm{~K}$ bytes. A 12 -bit $\mathrm{A} / \mathrm{D}$ converter is at tached to a DMA channel for digitizing spike waveforms (maximum resolution of 25 microsec). A Tektronix graphics terminal (Model 4006) and a hard-copy plotter are used to display the current status of the experiment. Experimenter communication with the system is through a Teletype Model 43 operating at 300 baud.

Mass storage for collected data and executable programs is implemented by the CLIPR remote file system. The remote file system allows remote laboratory computer systems' use of CLIPR Sigma 3 files as if they were directly connected to the remote microcomputer. Use of the system involves assembly language programming by way of a cross-assembler on the Sigma 3, until the PASCAL compiler is ready.

\section{PLANNING}

In the sections below, considerations made and problems overcome in development and implementation of CLIPR RITs are discussed. First, we address one of the coequal components in development and implementation: planning. Planning begins with specification of the application and progresses into a combination of hardware and software specifications. These processes are deeply influenced by the context in which they occur. Our point of view is perhaps conditioned to a major degree by the CLIPR central computer facilities. Other considerations may come into more important play in other environments.

The final major section of the paper focuses on actual implementation of the system, covering a range of general topics, from hardware implementation of the RIT, host and RIT software, and development tools.

\section{Application Specification}

Clear precise specifications of functional requirements and functional design of the application are critical to success of both planning and implementation phases of a project. Application specifications too general or ambiguous make the hardware and software specifications more difficult to derive. Ambiguous application specifications tend to introduce problems in implementation since they are subject to different interpretations and assumptions, according to context. One special problem is often encountered in context of computer-sophisticated environments: Hardware or software concerns may tend to determine the application specifications. This problem can best be dealt with by focusing on very clear, definite, written application specifications. If complete detailed specifications cannot be produced, a range of restrictive conditions and parameters will provide at least some protection and assistance.

One method of developing detailed specifications of the type needed is to describe the experimental procedures in terms of functional processes. The concept of a process is analogous to that in electronics of a "black box" device. The process may be described as follows: the data presented to the black box (input), what the box does with or as a result of the data (action), and the data that come out of the black box (output). This may be represented as follows:

\section{INPUT $\rightarrow$ ACTION $\rightarrow$ OUTPUT}

Input. For each possible input from the experimenter or experimental environment, the following characteristics, where applicable, should be developed in detail: (1) initialization input (e.g., random number seed, subject identification, etc.), (2) experimental event observation and recording (e.g., experimental events, latencies, accuracy required, frequency of event occurrence, etc.), (3) experiment control (e.g., abort experiment, change experiment parameters, control input such as pushbutton or terminal, etc.).

Action. Actions are described by stating what is to happen for each of the possible inputs. For example, if the input is a pushbutton response, then the associated action might be to record a response latency and output feedback to the subject.

Output. Each output should be described in terms of what it is or means and the form required. Outputs can generally be classified as (1) experiment status feedback, (2) experimental environment control output, and (3) saving of data.

Time-critical relationships. Each input that is associated with an action, producing an output, requires an additional piece of information: the critical response 
time. This is the maximum amount of time that can elapse after an input before the event associated with it is no longer relevant. For example, the response time for an experiment-initialization input would be acceptable at 5 or even $20 \mathrm{sec}$, but not at $30 \mathrm{~min}$. Likewise, if the action associated with an event is to record a current biophysical variable of the subject (e.g., heart rate), and the system response time for performing the measurement is $10 \mathrm{sec}$ or even $2 \mathrm{sec}$, then the measurement may not be relevant. A maximum response time should be specified for each input, action, and output relationship.

\section{Hardware}

In development of a plan for hardware to automate research in psychology, the issues that seem always to be present are feasibility and standardization. Although the issues are complex and interwoven, we have attempted to simplify and clarify at the risk of appearing to neglect important points.

Feasibility. There are five factors determining the level of feasibility in automating psychological research in a specific situation. These factors played an important part in the specific choices that we made in development of the CLIPR RITs described above. These factors are (1) cost, (2) technical capabilities of personnel on a project, (3) match of application specifications with available hardware, (4) packaging, and (5) support tools available.

Cost. This is perhaps the most obvious factor. However, since it is not a technical matter, we will not dwell on it here. Other feasibility factors influence cost, and the relationship is usually obvious. Where this is not true in our discussion, the relationship is pointed out.

Technical capabilities of project personnel. Realistic assessment of project staff and management is fundamental to success of the project. If a staff trained and experienced in digital electronics design and implementation is not available, the options available are narrowed. However, if engineering staff are available, then it becomes realistic to consider implementing custom electronics as a part of the automation facilities. As the microprocessor industry grows, so does the range of devices, controllers, and interfaces that are available to plug into commercially available "packaged" systems. Consequently, the level of technical competence needed in hardware implementation is decreasing. Generally, this development applies, at this time, to simpler systems and not to high-capability systems. For example, in planning for a general-purpose RIT to handle multiple, asynchronous subjects, with potential for high-demand numerical computation tasks, high data rate applications, and so on, we were led to select a computer that was not at the time represented among the available packaged systems. Consequently, the technical personnel requirements played a very important role in assessing the feasibility of implementing our own microcomputer RIT system with the Texas Instruments 9900 microprocessor. Since we have a high level of technical capability at CLIPR, our choice was justifiable.

The match between processor capabilities and application requirements. For a large portion of psychological research, most general-purpose microprocessors currently available are appropriate automation devices. However, the requirements imposed by application specifications must be checked carefully against processor capabilities or the developer runs the risk of ending up with a system that is incapable of doing the work, or one that will report erroneous results as a consequence of the inadequacies of the system. Perhaps a crude rule of thumb should be that if electromechanical and solid state programming devices have been used in the target class of experiments, then most available microprocessors possess the inherent capability of executing programs that will exceed the standards of the original equipment. However, where applications with high data rates, multiple asynchronous processes, intermixed arithmetic and control processes, and so on, are involved, the computational speed and technical capability of the processor must be investigated in detail to determine its appropriateness.

Properties not directly associated with processor instructions and speed may play an important role in assessing logical feasibility. Chief among these are considerations such as memory word ( 8 bit, 16 bit, etc.), register structure and operations (indexed addressing, register-to-register operations, etc.), interrupt structures and operation, memory address generation, memory field lengths, and so on. Evaluation of a system is complicated by interactions between the fundamental properties of the processor and the way that those properties are used (principally by software, but also by other hardware). As a consequence, evaluation of the basic functional elements of a microprocessor cannot stand alone, but it serves as a first step in a more elaborate procedure. If a packaged system of hardware and software is involved, the performance of the system will often be a better reflection of the adequacy of the software than of the hardware-and let the buyer beware, a fine processor can be poorly used. The hardware analysis cannot stop at the level of the processor. Very serious consideration of auxiliary and peripheral equipment to be included in the system must be made. Initially, the simple availability of the interfaces and peripheral equipment to perform such tasks as serial $\mathrm{I} / \mathrm{O}$, parallel $\mathrm{I} / \mathrm{O}$, and local storage management (disk, cassette, etc.) is fundamental to a feasibility analysis. And, here again, it is unfortunate that simply finding that Company $\mathrm{X}$ makes a device to perform $Y$ on systems supplied by Company $Z$ will not do. It is up to the planner to ascertain whether a device is compatible with the other equipment that will be installed in the microcomputer chassis, whether it 
uses the facilities of the central processor in a way that is compatible with the other equipment and with the application requirements, and so on. In the real-time domain, it is necessary to recognize that most of the devices, their interfaces to the processor, and software to drive these are designed and implemented in the world of "personal" computing and quasibusiness or quasiscientific computation. As a consequence, those systems may not be compatible with the more demanding or complex applications in experiment automation.

Packaging. The types of packages that can be obtained on the commercial microprocessor market today are vastly more well developed than only 2 years ago. However, care is required in evaluating the packaging of systems. In completely off-the-shelf systems, even the seemingly obvious things such as the number of printedcircuit cards that can be installed in the chassis/bus of the system must be evaluated carefully to determine the feasibility of using the system in the proposed application. This interacts, of course, with the subsystems that are used with a package system and the number of chassis slots that are used by the subsystems. The mundane consideration of the number and size of cables that are needed to connect to external devices has been known to seriously affect cost and level of technical capability needed to complete a project.

The cost of packaged systems must be evaluated carefully. Unless the vendor is able to provide all of the necessary subsystems, peripherals, and so on, they must be priced separately with consideration of the cost of any work and programming needed to add them. Mixing components from various vendors also carries with it the hazard of subtle incompatibilities that can be costly to detect, correct, or circumvent. For example, certain memories will have timing properties that make them hazardous for the user within certain configurations of bus and microprocessor.

A word of caution to real-time users of packaged microprocessor systems is in order. Developers and vendors of such systems generally do not have direct experience with real-time applications, multiuser asynchronous applications, and so on. A great deal of care in using such systems in real-time is called for. Again, the most significant part of the interaction between a vendor and a buyer is what the vendor does not say, rather than what the vendor does say. Unless the vendor can demonstrate that a packaged system does satisfy the functional requirements of a given application, with actual measured performance, it is most responsible for the scientist to assume that the system cannot satisfy those requirements until the demonstration can be made.

In custom-designed and -fabricated systems, considerations of packaging also play a role. However, in this case the issue of feasibility is resolved in cost considerations and the competence of the staff available to implement the system. In this case the cost of personnel becomes a very major item. The availability of qualified technical personnel outside of specific funding for a project sometimes makes this route more attractive, but use of commercially available components is the most easily defended use of resources. In undertaking special development, time factors also become critical. Also, the developer should take into account software development factors, as well as hardware factors.

Support. In developing a plan to implement a realtime microprocessor system, the issue of the support available to execute the plan is directly related to the competence question and to the cost question. Extensive tools are available on certain systems to assist in the development of both hardware and software components of a system. If packaged systems are not a feasible option in a given application, and it is necessary to develop a system, the amount of aid available and its cost must be an important component of a plan to develop the system.

Even if a packaged system is available, satisfying the application requirements, the critical question of programming development and system maintenance (both hardware and software) must be carefully analyzed before final feasibility can be determined for using a specific system for a particular application. Software support of development is discussed extensively in a section that follows. Also, the use of development tools has a prominent place in our discussion of implementation (below).

Standardization. This is a tricky issue, and one that is often responded to on the basis of feelings that one should resist attempts to restrict freedom. However, in the real world of finite, if not restricted, resources and knowledge, standardization of methods and instrumentation is a potential source of flexibility and range of application. In a multiplc-system environment with $\mathrm{N}$ different processors, $\mathrm{N}$ different assembly languages, $\mathrm{N}$ different interface systems, and so on, the difficulty of maintaining and exploiting the capabilities of the $N$ systems is probably an exponential function of $\mathrm{N}$. Consequently, the research productivity of $\mathrm{N}$ systems becomes something on the order of the inverse of the exponential function of $\mathrm{N}$.

Standardization procedures have resulted in a wide variety of standards being adopted in this country and abroad, covering such diverse products as programming languages, communication protocols, connectors, use of conductors in a computer bus, character code definitions, and so on. If these standards are adhered to by a vendor in a packaged system or by a developer in a custom system, the entire enterprise of automating research becomes simpler and more satisfactory. It is highly desirable to insist in planning and implementation of real-time systems that recognized standards be adhered to in all aspects of the system, as far as those standards are relevant and applicable to the application being automated.

Standardization of hardware, including optional 
modules, and software within an administrative unit like a psychology department has the advantage of controlling the number of compilers, assemblers, device interfaces, development aids, and so on, that must be maintained, taught, and used within that unit. The efficiencies of the standardization are very significant. On the other hand, if the coordination and standardization within the unit is sensitive to the full range of application needs represented, the potential for restriction of research and development of technology can be minimized.

\section{Software Specification}

After the application has been clearly specified and a hardware configuration proposed, the circle must be closed to insure correspondence between the experiment requirements and the functions provided by the proposed hardware configuration. This closure is generally within the domain of software. Generating software specifications requires analysis of the hardware and application to determine both the functional software models required to implement the application and the tools necessary to construct the application programs.

Identification of the major functional software modules requires a careful analysis of the application specification for purposes of mapping it into an equivalent specification using computer concepts. For example, an experimenter's concept of response latency must be mapped into the computer concept of a 32-bit millisecond-clock value. This analysis should be performed by a person trained in computer programming and familiar with the hardware specification. There generally will be one module corresponding to each process identified in the application specification.

Once the major functional modules have been identified, the software tools that are required can be determined. The minimal tools and their relationships are shown graphically in Figure 4. Although the tools

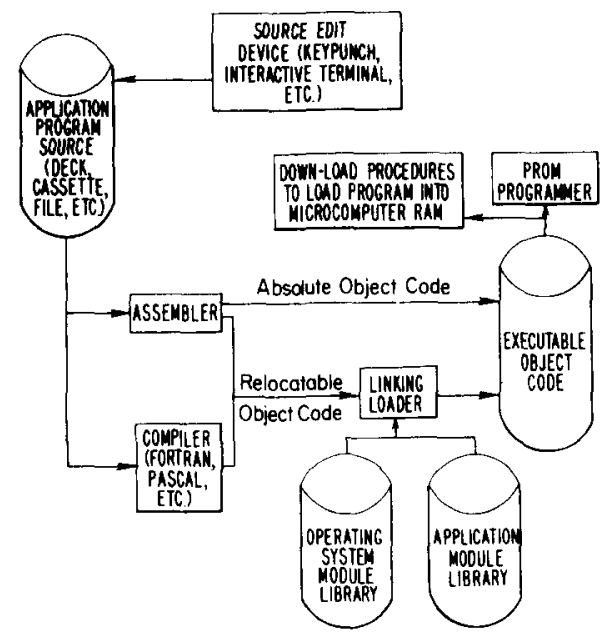

Figure 4. Software tools and their relationships.

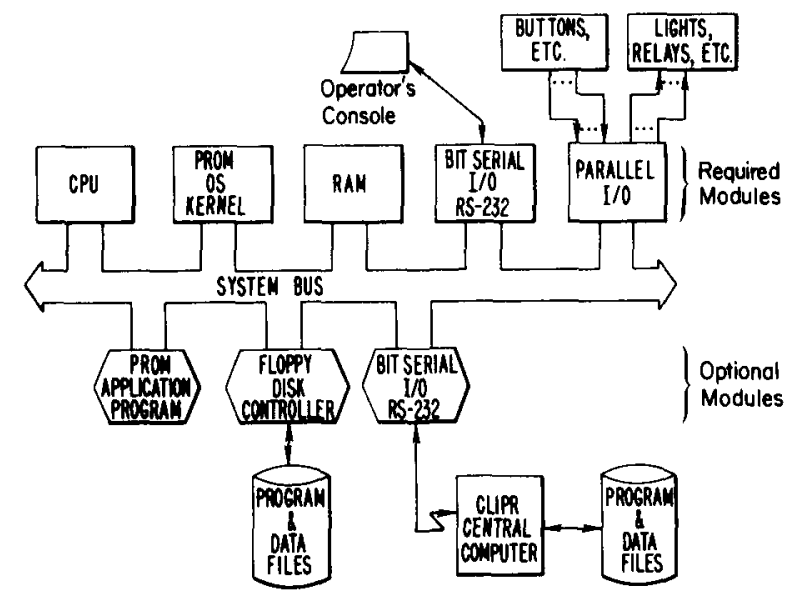

Figure 5. An illustrative microprocessor RIT system.

diagrammed do not represent an absolute minimum set, they do represent the minimal usable set for general applications (and systems) development.

These tools include program development tools or "development system software," as well as applications and systems software module libraries.

Development system software components consist of implementation language translators (assemblers and compilers), linking loaders, library facilities, source language storage, and editing facilities. Systems libraries contain software modules that provide operating system functions, such as input and output control, timing routines, and implementation language support routines such as floating-point arithmetic routines.

Applications libraries contain software modules that are specific to a class of applications. For example, all applications programs for the video processor described above would utilize a common set of software modules that implement various primitive functions on the video processor.

For medium- and large-scale applications programs, the most critical tool is the implementation language that is available to the programmer. In almost all systems, some form of an assembly language and its translator are available. Less frequently, the application programmer will have access to a higher level language compiler (e.g., BASIC, FORTRAN, or PASCAL) for the specified target microcomputer system. The range of applications for which each of these languages is suited is restricted by: the language itself, the implementation of the language, and the library routines available to programs written in that language.

Figure 5 is an attempt to illustrate how a particular hardware and software system might be implemented, in logical form. Not all of the modules shown in Figure 5 are required in every system. The required modules are: CPU, some RAM, some PROM, ROM or EPROM, a serial interface for the operator's console, and some number of parallel interface $\mathrm{I} / \mathrm{O}$ bits. Actually, the last two listed elements are not both required, but since the cost of each of the two modules is so modest (e.g., 
about $\$ 30$ on a typical system), the minimal target system configuration can easily incorporate both. The serial interface and operator's console would, of course, be needed unless the target system were programmed on another machine, with the basic operating system and applications program loaded into ROM by procedures outside of the target system.

The optional modules are usually specialized I/O interfaces for equipment required by the application, such as local floppy disk systems, network interfaces, real-time clocks, and so on.

\section{IMPLEMENTATION}

Although hardware and software considerations are intricately interwoven, we develop the discussion here in separate sections. The reader is cautioned that satisfactory development in one domain does not imply a similar situation in another.

It would be most desirable to have a catalog of pieces and parts of equipment or systems, and a library of software tools and application modules. This situation does not exist. The technician, student, or researcher who wants to develop a microprocessor-based laboratory system must become well versed in the technology of hardware and software to succeed. It is easy to be misled by the blandishments of packaged system vendors. Most of what they say is probably true. However, what is not said is the key to full understanding of what is really involved. And, unless one knows how to fill in the missing elements in a system description and know what must be done to supply those elements, it is easy to be misled.

\section{RIT Hardware}

In this section, we sketch briefly some details of our experience at CLIPR in developing the Motorola 6800 (Motorola Semiconductor Products, Inc., 1975, 1976a, 1976b) and Texas Instruments 9900 (Texas Instruments, Inc., 1976a, 1976b, 1976c) hardware systems as RITs. Figures 6 and 7 represent the structures of these two systems.

Selection of the 6800 violated many of the guidelines

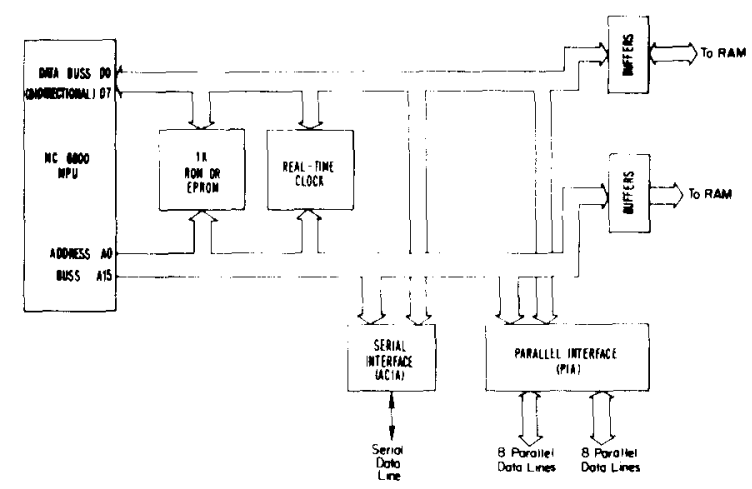

Figure 6. CLIPR system based on the Motorola 6800.

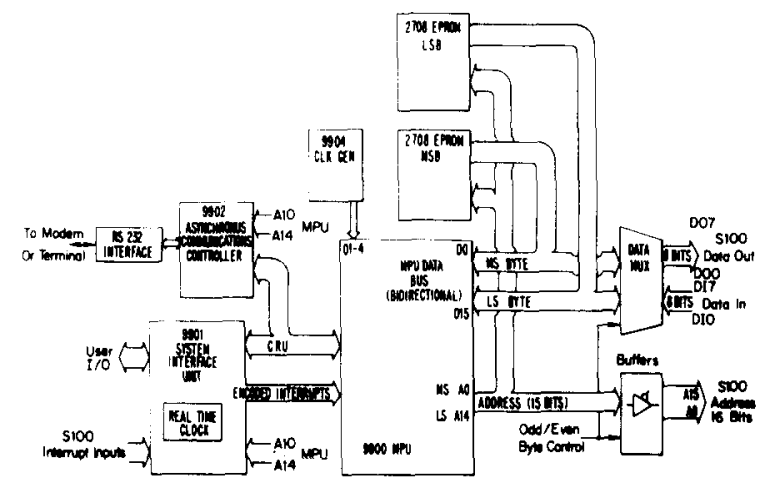

Figure 7. CLIPR system based on the Texas Instruments 9900.

we now propose. It was adopted at first largely because it was available. It turns out not to have been a poor choice, largely because almost any microprocessor would have been appropriate for the applications that we assign to the 6800 systems.

Selection of the 9900 was more careful and deliberate. We attempted to find the combination of available hardware and software that would best meet all of our anticipated needs, including high data rates, multiple asynchronous tasks, network controller, intermixed numerical and real-time components, and so on. When we started our investigation, the hardware could be divided into four categories: chips, single-board computers, (e.g., Motorola Exorcizer and Intel SBC), systems that interfaced to the S100 bus (Goble, 1977; Morrow \& Fullmer, 1978), and development systems offered as packages. Because of cost and the lack of suitable interfaces, the single-board computers and development systems were dropped from consideration. That left the microprocessor chips and the S100 bus systems. None of the 8.bit processors appeared to handle the applications anticipated at the high end of the demand range. Furthermore, the low end of complexity was being covered by the default-selected 6800. Certain systems such as those offered by Data General (MicroNOVA) and Digital Equipment Corporation (LSI-11) were considered but rejected because of inadequate computer power and high cost. The remaining option was the 9900 . In addition to the $16-$ bit memory on the 9900 , we liked a number of features: (1) The processor was available on a single chip, making it usable in very inexpensive controller devices, (2) register structure and addressing superiority, (3) hardware arithmetic facilities, (4) powerful interrupt structure, and (5) the promise of a wealth of software (which has not yet been fulfilled). The 9900 appeared to involve a greater degree of cost than other processors, if we were to limit our system development to devices offered by Texas Instruments in the 990 family of computers based on the 9900. However, other 16-bit systems (Data General and Digital Equipment Corporation, primarily) involved greater cost, without the option of using the 
processor chip in smaller implementations. Several fundamental programmed operations (memory-tomemory transfer of arrays, code translation with table look-up, interrupt handling, etc.) were hand coded in the assembly language of several target machines (Intel 8080, Motorola 6800, MOS 6502, Texas Instruments 9900). Some of the programs were written in a reentrant mode. Execution time for the code was calculated using instruction times. The 9900 did as well or better than other processors in this study. In certain instances, involving arithmetic and interrupt handling, particularly, the 9900 excelled. Other comparisons, such as memory (number of bytes) required for the programs, ease of coding, and so on, favored the adoption of the 9900 .

The minimum packaged system available with the 9900 at the time we made the selection was a $\$ 10,000$ development system. The cost of this system was too high to consider it as a standard for our general-purpose RIT. Furthermore, the packaging was not attractive, in view of the availability of a growing amount of hardware and software based on other processors. And, the bus for the development system from Texas Instruments was not compatible with the 6800 . Consequently, we investigated the possibility of selecting a bus that would permit a two-level implementation of RITs: one level with the 6800 and a second with the 9900 . Use of a single bus permits a greater degree of standardization of RITs. Also, this strategy permits integration of off-theshelf system components into the laboratory.

One bus was prominent in the industry: the S100. The S100 bus has a number of advantages: (1) It is well documented (Conway, 1977; Goble, 1977; Morrow \& Fullmer, 1978); (2) it has been widely adopted by systems developers; (3) a large number of devices plug into the bus; and, (4) the data structures provide a reasonable set of signals, data paths, and address space. On the other hand, the versatility of the bus has created numerous compatibility problems (Cushman, 1978). Attempts are being made to standardize the $S 100$ bus (see, especially, Morrow \& Fullmer, 1978). In the meantime, the "almost" standard bus poses problems for the nontechnical user. Although the flexibility allows a developer to configure systems using the bus in just about any way desired, many of the so-called S100compatible interfaces are only partially compatible and have serious flaws in certain configurations. Often, these flaws are subtle and can lead to disastrous problems for the user. Another problem has been the inconsistent use of pins on the bus, making it impossible to adopt certain combinations of devices designed to be used with the S100.

The Motorola 6800-based RIT has become a quick solution to instrumentation problems; four such systems are currently in use at CLIPR. Relatively small amounts of manpower and time are spent in delivering a fully functional 6800 RIT to a laboratory. As might be anticipated, the greatest area of cost is in software development for a specific application. The hardware cost of a RIT configuration like that shown in Figure 6 (minimum configuration) is approximately $\$ 350$, without keyboard/display, modem/coupler, or other peripheral systems.

The 9900 is a 16-bit machine and the $\mathrm{S} 100$ bus has an 8-bit data path. This seeming incompatibility is resolved by providing a multiplexing scheme on the CLIPR 9900 central processor board that results in two fetch or store operations per normal memory access cycle of 524 nanosec. The memory used is 250 -nanosec memory, which is only $15 \%$ to $20 \%$ more expensive than the 500-nanosec memory normally used with the 9900 . The same property of the 9900 posed another problem: memory-mapped I/O (Townsend, 1976). Device registers can change during a 2-byte transfer, causing data loss and error. The solution of this problem involves allocating a portion of the memory address space for memory-mapped I/O and providing the $9900 / \mathrm{S} 100$ interface with special functions to coordinate the 16-bit and 8-bit operations.

A specific example of implementation problems involving peripheral devices is perhaps useful at this point. In attempting to find a floppy disk drive interface and software for the CLIPR 9900 system, we have had difficulty in finding a packaged disk system for the 9900 that permits DMA. Furthermore, the systems that we have investigated work by locking out the central processor until $\mathrm{I} / \mathrm{O}$ is complete, or by generating interrupts on a sector-by-sector basis when seeking the target sector. Either of these schemes is satisfactory in low-demand applications. However, in our target application with asynchronous processes (multiple subjects, multiple experiments), these $\mathrm{I} / \mathrm{O}$ driver schemes render real-time responsiveness and time-critical processes impossible as coexisting processes with the disk system.

The result of our work is a single printed-circuit board that plugs into the $\mathrm{S} 100$, with all of the special (custom) features of the central processor located on that board. All other boards are standard S100. compatible boards, including memory and device interfaces and controllers. The 9900 operates at full speed.

The full detail of the CLIPR microcomputer, using the 9900 , will be spelled out in other publications being planned. The cost of this development has been rather high, although it involved primarily personnel resources that were not incremental costs to CLIPR. We estimate that the cost of a CLIPR microcomputer central processor will be on the order of $\$ 350$ with all components, including the 9900 microprocessor, installed. That price does not include bus, memory, power supply, interfaces, and so on. Adding a chassis/ bus with power supply, $8 \mathrm{~K}$ bytes of memory, and a keyboard/display to make up a minimally usable system brings the total cost to slightly less than $\$ 2,000$. At this time CLJPR has five 9900 systems in operation or under 
construction, ranging in cost from the basic $\$ 2,000$ to a grandly developed system with plotter, graphic display, and so on, for $\$ 10,000$.

Development of the CLIPR microprocessor systems would have been greatly simplified and accelerated had we been able to purchase full-blown hardware/software packages. Such packages, with specialized facilities such as those for programming PROMs, are useful in building and checking both hardware and software being developed and are called "development systems." There are several development systems commercially available. Some of these are as follows: The Intel system supports the development of its 8000 series CPUs; the Tektronix 8002 system supports the development of systems based on, among others, the Intel 8000 s, the Texas Instruments 9900, and the Morotola 6800 CPUs; and the Texas Instruments system supports development of the 9900 CPU only.

\section{Host Hardware}

As long as appropriate software on a host is available, the detailed specification of host computer hardware is of little significance. However, the overall implementation of a host-RIT system requires several elements that might be considered host hardware: (1) sufficient storage for RIT programs and data sent from the RIT, (2) communications equipment coordinated with those of the RIT, and (3) responsiveness to requests from the RIT for services. The primary effect on implementation of a RIT when the host is inadequate in one or another of these areas is expansion of RIT memory, both central and peripheral.

At CLIPR we have been concentrating to this point on the development of individual RITs, with CLIPRNET and RITS waiting for completion of current development of the CLIPR 9900 RIT hardware/software systems.

\section{Software}

The software tools provided with a development system are most desirable when attempting to develop a real-time microcomputer system. However, commercially available development systems are still in developmental stages, without all of the tools needed for real-time programming, or even systems work.

As desirable as a development system may be, budgetary considerations may prohibit acquisition of one system to develop the second system. Under such circumstances, the developer must proceed to develop those portions of the development system that are minimally sufficient (or better) to do the job. New microprocessor systems can be developed without a development system, but much more personnel time is involved. The first step is to procure, modify, and/or write and test an assembler, loader, library, PROM programmer, and possibly a high-level language compiler.

We do not have a vendor-supplied development system at CLIPR. Consequently, a great deal of effort has been spent in procuring, modifying, and writing necessary software tools. First, a 9900 cross-assembler was obtained from Texas Instruments. It was written in FORTRAN for a 32-bit word machine and modified to run on the University of Colorado's CDC6400, a 60-bit word machine. There was no loader available, so we wrote a linking loader that accepts the assembler relocatable output and library input. In conjunction with this project, we built a Cramer PROM programmer kit (Cramer Electronics, Inc., 1976), interfaced it to the Sigma 3, and wrote a Sigma 3 driver program to control the actual PROM programming. Our bootstrap program for the 9900 RIT is a modified version of TIBUG, a Texas Instruments product for a single-board computer. Texas Instruments has now released a complete cross-support package, written in FORTRAN for a 16-bit word machine. This package includes assembler, linking loader, simulator, and debugger, but we chose to implement only the assembler on the Sigma 3, since we had obviated the need for or could not use the rest of the new package. In our case, reworking the assembler for the Sigma 3 was justified, as the Sigma 3 is CLIPR's computer, and we must buy time on the CDC6400.

Since we hope that only the RIT cold-start bootstrap, network communications, and $\mathrm{I} / \mathrm{O}$ control will be written in assembly code, we are presently working on a Sigma 3 PASCAL cross-compiler for the 9900.

Currently, several of our RITs contain 6800 s. To support this development, we have a Motorola absolute assembler that was modified to run on the Sigma 3

Presently, our software is assembled, compiled, loaded, and, where appropriate, programmed into PROMs and EPROMs using the Sigma 3. Some RIT monitor modules and user-application programs are not resident in ROM, but are loaded into RAM from an external source as needed.

Each RIT is not necessarily designed to support its own software development, or the storage of its own transient program modules, which are to be loaded into RAM as needed, or the experimental data collected. Therefore, before any RIT software development could be done, we needed a communications package with a file system available to every RIT on the network. In order to have individual RITs operational as soon as possible, we use serial transmission over voice-grade telephone lines, with a minimal protocol, as the communications between host and RIT. A remote file system, written in FORTRAN, is currently running on the Sigma 3 host computer. RIT program modules can now be stored on disk files or other devices accessible to the Sigma 3 and sent to a RIT, upon request to the remote file system, over available communication lines. File transfer goes both ways (hostRIT and RIT-host) in order to accommodate RIT 
program downline loading from the host to the RIT, host storage of data collected by the RIT, other data generated on the host but used by the RIT, and so on.

\section{SUMMARY}

It has become common to hear opinions that suggest that throwing a microprocessor at a laboratory instru. mentation problem will solve the problem. This is most emphatically not true without heavy qualification. The great reduction in cost of computer equipment has tended to place such equipment in more and more laboratories. However, other factors are not changing as fast as the cost of hardware. Training of psychologists in computer technology is primitive. Software tools to use computers are primitive. Training of psychologists to use (not to mention create) software tools is primitive. And the costs of personnel and tools necessary to make microprocessors effective laboratory instruments are increasing and out of the range of feasibility for a host of new microprocessor owners. For people who went through the early development of on-line and real-time computer automation of psychological research, there is a horrifying déjà vu experience in watching people thrash away at problems with microcomputers that were faced 10 to 15 years ago with minicomputers. It seems a pity that so many people are going through a painful process that should be buried in the past by all the lessons learned. Thus, we close with a benediction: Go thus out and get thyself trained and acquire unto thyself tools worthy unto the task.

\section{REFERENCES}

BaILEY, D. E. The Computer Laboratory for Instruction in Psychological Research, CLIPR. Behavior Research Methods \& Instrumentation, 1972, 4, 95-96.

BAILEY, D. E. Computer training in psychology at the University of Colorado. Behavior Research Methods \& Instrumentation, 1973, 5, 189-191.

Bailey, D. E., \& Polson, P. G. Real-time computing in psychology at the University of Colorado. American Psychologist, 1975, 30, 212.218.

Barma, A., \& PoRAt, D. I. Introduction to microcomputers and microprocessors. New York: Wiley, 1976.

BIBBERo, R. J. Microprocessors in instruments and control. New York: Wiley, 1977.

Colorado Video, Inc. Instruction manual, Model 270A video digitizer. Boulder, Colo: Author, 1977. (a)

Colorado Video, Inc. Instruction manual, Model 275 video expander. Boulder. Colo: Author, 1977. (b)

CONWAY, J. CPU boards-A matter of bits, buses and BASIC. EDN, November 20,1977, p. 144.

Cramer Electronics, Inc. PROM programmer Cramerkit reference manual. Newton, Mass: Author, 1976.

Cushman, R. H. Microcomputer bus standardization-Will designers' needs be met? EDN, February 20, 1978, p. 97.

Goвle, W. M. Introducing the S100 standard small computer bus structure. Interface Age, June 1977, p. 68.

Morrow, G., \& Fullmer, H. Proposed standard for the $\$ 100$ bus. IEEE Computer, 11(5), 84-90, 1978.

Motorola Semiconductor Products, Inc. M6800 microcomputer application manual. Phoenix, Ariz: Author, 1975.

Motorola Semiconductor Products, Inc. M6800 microcomputer design data. Phoenix, Ariz: Author, 1976. (a)

Motorola Semiconductor Products, Inc. M6800 microcomputer programming manual. Phoenix, Ariz: Author. 1976. (b)

OSBORNe, A. An introduction to microcomputers: Basic concepts (Vol. 1). Berkeley, Calit: Osborne, 1976. (a)

Osborne, A. An introduction to microcomputers: Some real products (Vol. 2). Berkeley, Calif: Osborne, 1976. (b)

Polson, P. G. Microprocessors: Their impact on real-time computing in psychology. Behavior Research Methods \& Instrumentation, 1978, 10, 139-147.

SOUCEK, B. Microprocessors and microcomputers. New York: Wiley, 1976.

TEXAS INSTRUMENTS, INC. 990 computer family systems handbook. Houston, Tex: Author, 1976. (Document No. 945250-9701) (a)

TEXAS Instruments, InC. TMS 9900 microprocessor data manual. Houston, Tex: Author, 1976. (b)

TEXAS INSTRUMENTS, INC. TMS 9900 system development manual. Houston, Tex: Author, 1976. (Bulletin MOSA2) (c)

Townsend, M. Memory mapped I/O for the TMS 9900 . New Electronics, 19(22), 19-22, 1976. 